\title{
Destroying the event horizon of regular black holes
}

\author{
Zilong $\mathrm{Li}$ and Cosimo Bambi* \\ Center for Field Theory and Particle Physics \& Department of Physics, Fudan University, 200433 Shanghai, China
}

(Dated: August 19, 2018)

\begin{abstract}
Recently, several authors have studied the possibility of overspinning or overcharging an existing black hole to destroy its event horizon and make the central singularity naked. When all the effects are properly taken into account, any attempt to destroy the black hole seems to be doomed to fail, in agreement with the weak cosmic censorship conjecture. In this letter, we study the possibility of destroying the event horizon of regular black holes. These objects have no central singularity and therefore they are not protected by the cosmic censorship hypothesis. Our results strongly support the conclusion that regular black holes can be destroyed. If we believe that the central singularity in astrophysical black holes is solved by quantum gravity effects, we might have a chance to see the black hole's internal region and observe quantum gravity phenomena. As our finding implies the violation of the black hole's area theorem, the collision of two black holes may release an amount of energy exceeding the Hawking bound, which can be experimentally tested by gravitational wave detectors.
\end{abstract}

PACS numbers: 04.70.Bw, 04.20.Dw

Under the main assumptions of the validity of the strong energy condition and of the existence of global hyperbolicity, in general relativity the final product of the collapse is a singularity of the spacetime [1]. At the singularity, predictability is lost and standard physics breaks down. According to the weak cosmic censorship conjecture, singularities produced in the gravitational collapse must be hidden within black holes and cannot be seen by distant observers 2]. The validity of this conjecture is still an outstanding open problem in gravitational physics. While it is thought to be correct at some level, we also know physically relevant counterexamples in which naked singularities can be created from regular initial data [3]. The validity of the weak cosmic censorship conjecture is a fundamental assumption in the theory of black hole thermodynamics. On the other hand, its possible violation would allow to observe high curvature regions, where new physics is expected to show up.

Some authors have studied the possibility of violating the weak cosmic censorship conjecture by destroying an existing black hole [4 8]. The advantage of this set-up is that we do not need any assumption about the matter content, which is the crucial and questionable point in all the studies on the validity of the weak cosmic censorship hypothesis. In 4-dimensional general relativity, a black hole is described by the Kerr-Newman solution and it is specified by its mass $M$, spin $J$, and electric charge $Q$. The condition for the existence of the event horizon is

$$
M^{2} \geq Q^{2}+a^{2},
$$

where $a=J / M$ is the spin parameter. When Eq. (1) is violated, there is no horizon and the central singularity is naked. One can thus imagine an experiment in which a black hole absorbs a small particle of energy $E$, angular

\footnotetext{
* Corresponding author: bambi@fudan.edu.cn
}

momentum $L$, and electric charge $q$. If the new object violates the bound (1), the small particle has destroyed the black hole: the event horizon disappears and the central singularity becomes naked.

To see if it is really possible to destroy a black hole, we need to check if the small particle with $E, L$, and $q$ can actually be absorbed by the black hole. In the test particle approximation, one can follow Ref. [4] and finds that the absorption condition is

$$
E \geq \frac{q\left(A_{\phi} g_{t \phi}-A_{t} g_{\phi \phi}\right)-g_{t \phi} L}{g_{\phi \phi}},
$$

where $A_{\mu}$ is the 4-potential of the electromagnetic field and Eq. (2) must hold from the point where the particle is fired to the black hole's event horizon, where the particle is absorbed. If Eq. (2) does not hold, it simply means that the electric charge and/or the angular momentum of the test particle are too high and the particle cannot reach the event horizon. The overcharging/overspinning condition is instead

$$
(M+E)^{2}<(Q+q)^{2}+\left(\frac{a M+L}{M+E}\right)^{2} .
$$

We can thus consider a near extremal black hole and find for which values of $E, L$, and $q$ both Eqs. (2) and (3) are satisfied; that is, the particle can be absorbed and the new state has no event horizon. In the KerrNewman spacetime in Boyer-Lindquist coordinates, the non-vanishing metric coefficients are

$$
\begin{aligned}
& g_{t t}=-\left(1-\frac{2 m r}{\Sigma}\right), \quad g_{t \phi}=-\frac{2 a m r \sin ^{2} \theta}{\Sigma}, \\
& g_{\phi \phi}=\left(r^{2}+a^{2}+\frac{2 a^{2} m r \sin ^{2} \theta}{\Sigma}\right) \sin ^{2} \theta, \\
& g_{r r}=\frac{\Sigma}{\Delta}, \quad g_{\theta \theta}=\Sigma,
\end{aligned}
$$

while the 4-vector of the electromagnetic field is

$$
A_{t}=-\frac{Q r}{\Sigma}, \quad A_{\phi}=\frac{Q r}{\Sigma} a \sin ^{2} \theta
$$


where

$$
\Sigma=r^{2}+a^{2} \cos ^{2} \theta, \quad \Delta=r^{2}-2 m r+a^{2},
$$

and $m$ is given by

$$
m=m_{\mathrm{KN}}=M-\frac{Q^{2}}{2 r} .
$$

In Ref. [5], the authors find that a small particle can destroy a black hole. However, these studies ignore the radiated energy as well as the particle's self-energy. The former makes easier the destruction of the black hole, while the latter makes more difficult the absorption of the particle, as it may introduce a turning point before the particle reaches the black hole's event horizon. While there is not yet the theory and the technology to perform complete calculations, analyses beyond the test-particle approximation strongly suggest that this is indeed the case and the self-energy acts as a cosmic censor 9, 10.

The validity of the weak cosmic censorship conjecture seems thus to be confirmed by these gedanken experiments when all the effects are properly taken into account, and it is apparently impossible to destroy an existing black hole. However, we may guess that a real black hole has no central singularity, as the latter is more likely a pathological feature associated with classical general relativity, to be removed by (unknown) quantum gravity effects. If this is indeed the case, astrophysical black holes may not be protected by the weak cosmic censorship conjecture and there may be a chance to destroy their event horizon 11 .

While we do not have yet any robust and reliable quantum theory of gravity capable of telling us how the singularities in the interior of black holes are solved, in the literature there are some toy-models of black hole solutions without the central singularity. The prototype of these regular black holes is the Bardeen metric [12, which can be formally obtained by coupling Einstein's gravity to a non-linear electrodynamics field [13]. Another popular example is the Hayward black hole metric [14]. The rotating solutions of the Bardeen and the Hayward metrics have been obtained in Ref. 15. In the non-rotating case, these solutions violate the strong energy condition, but not the weak one. For spinning black holes, even the weak energy condition is violated, but such a violation can be made very small [15. In the rotating Bardeen and Hayward black hole spacetime, the metric coefficients $g_{\mu \nu}$ are still given by Eq. (4), but $m$ is now given, respectively, by

$$
m_{\mathrm{B}}=\frac{M r^{3}}{\left(r^{2}+g^{2}\right)^{3 / 2}}, \quad m_{\mathrm{H}}=\frac{M r^{3}}{r^{3}+g^{3}},
$$

where $g$ can be interpreted as the magnetic charge of the non-linear electromagnetic field or just as a quantity introducing a deviation from the Kerr metric and solving the central singularity. For neutral particles, the absorption condition is still given by Eq. (2). There is instead no simple formula like Eq. (1) for the overcharging/overspinning condition, so we have to check that the initial state is a black hole, i.e. the equation $\Delta=0$ (with the correct $m$ ) has at least one positive solution for $r$, and that for the final state $\Delta=0$ has no solutions.

Let us now compare the attempt to destroy a KerrNewman black hole with the one for regular black holes. Since the nature of the electromagnetic fields is different, a direct comparison is possible only considering the case in which the small particle has no charge. When we consider a near extremal Kerr-Newman black hol $\oint^{1}$ we find that the region of the values of $E$ and $L$ for which the small particle destroys the event horizon is very narrow. Two specific cases are reported in the left panels of Fig. 1. As noted in Ref. 7, 10, the allowed energy range of $E$ is of order $L^{2} / M^{3}$. In our cases, we find $\Delta E \sim 10^{-11}$, in perfect agreement with this estimate (here we have set $M=1$ and find $\left.L \sim 10^{-5}\right)$. The energy range of $E$ is of the same order (and opposite sign) of the expected corrections from the particle's self-force [7, 10. It is thus plausible that the region disappears when this effect is properly taken into account and that the particle's self-energy prevents the destruction of the black hole, confirming the validity of the weak cosmic censorship conjecture.

We can then repeat the same experiment for the Bardeen and Hayward black hole. Specific cases of the region for which the test particle can destroy the event horizon are reported in the central (Bardeen) and right (Hayward) panels of Fig. 1. The result is clear: now such a region is definitively larger than the one in the Kerr-Newman case. In the plots in Fig. 1 we have also distinguished the bound (blue-dark region) and the unbound (yellow-light region) orbits capable of destroying the black hole. Since the particle's self-force should here introduce a correction of the same order of the KerrNewman case, $\sim L^{2} / M^{3} \sim 10^{-10}$, while the allowed region is significantly wider, $\Delta E \sim 10^{-5}$, we may conclude that regular black holes can be destroyed.

It is even more interesting to note that it is probably not necessary an ideal experiment to destroy a regular black hole, but a common astrophysical phenomenon like the accretion process from a disk can presumably do the job. In this framework, the gas falls onto the black hole as it loses energy and angular momentum. In the case of a thin disk on the equatorial plane, the gas reaches the innermost stable circular orbit (ISCO) and it then plunges quickly onto the compact object, which changes its mass $M$ and spin $J$ by [16]

$$
M \rightarrow M+\delta M, \quad J \rightarrow J+\delta J
$$

where

$$
\delta M=\epsilon_{\mathrm{ISCO}} \delta m, \quad \delta J=\lambda_{\mathrm{ISCO}} \delta m
$$

\footnotetext{
1 To be more specific, we first fix $a$ and then we take $Q=$ $Q_{\text {extremal }}-\varepsilon$ with $\varepsilon=10^{-10}$. In the next paragraph we do the same for the regular black holes, replacing $Q$ with $g$.
} 
$\epsilon_{\mathrm{ISCO}}$ and $\lambda_{\mathrm{ISCO}}$ are, respectively, the specific energy and the specific angular momentum of a test-particle at the ISCO, while $\delta m$ is the gas rest-mass. Here, we still assume that the particles are neutral, so $Q \rightarrow Q$ or $g \rightarrow g$. Fig. 2 shows the evolution of our black holes as a consequence of the accretion process from a thin disk (red solid curves) for an initially non-rotating object and different values of the initial charge. In these numerical calculations, we have used $\delta m=10^{-6}$ or $10^{-7}$. The black dashed-dotted curves are the boundaries separating black holes from horizonless states (naked singularity in the Kerr-Newman case, some kind of regular solitons for the Bardeen and Hayward ones). As can be seen in the enlargement in the top right corner of every panel, in the case of the Kerr-Newman black hole, the red solid line cannot cross this boundary, even for high values of the electric charge $Q$, while that is possible for the regular black holes, even for relatively low values of $g$ (and it becomes more and more easy as $g$ increases).

Conclusions - Recently, there have been a lot of interest in the possibility of destroying the event horizon of a black hole to violate the weak cosmic censorship conjecture. In the test-particle approximation, it seems like there are some orbits for which a small particle can plunge onto the black hole and destroy its event horizon. However, the allowed range of the parameters of the small particle is very narrow and of the same order of the expected corrections from the particle's self-energy, suggesting that the latter prevents the destruction of the black hole if properly taken into account. In this letter, we have considered the possibility of destroying a regular black hole. As this object has no central singularity, it is not protected by the weak cosmic censorship conjecture. We have presented two different examples (gedanken experiment and accretion process from a thin disk) strongly suggesting that regular black holes can be destroyed. Unlike the Kerr-Newman case, the parameter region for which the test particle can destroy the black hole's event horizon is not narrow, while the expected correction from the self-energy is of the same order of the one in the Kerr-Newman spacetime. Moreover, it seems so easy to destroy such regular black holes that we do not need ideal experiments with particles of very specific $E$ and $L$, but the natural accretion process from a thin disk can do the job. Lastly, let us notice that our regular black holes violate the black hole's area theorem. For standard black holes, this theorem has important implications like the existence of an upper bound for the energy released in the collision of two black holes [17. In the case of regular black holes, such a bound does not exist and that may be tested by future detections of gravitational waves from the coalescence of two black holes.

\section{ACKNOWLEDGMENTS}

This work was supported by the Thousand Young Talents Program and Fudan University.
[1] S. W. Hawking and R. Penrose, Proc. Roy. Soc. Lond. A 314, 529 (1970); S. W. Hawking and G. F. R. Ellis, The Large scale structure of space-time (Cambridge University Press, Cambridge, UK, 1973).

[2] R. Penrose, Riv. Nuovo Cim. Numero Speciale 1, 252 (1969) [Gen. Rel. Grav. 34, 1141 (2002)].

[3] P. S. Joshi, Gravitational collapse and spacetime singularities (Cambridge University Press, Cambridge, UK, 2007); P. S. Joshi and D. Malafarina, Int. J. Mod. Phys. D 20, 2641 (2011) arXiv:1201.3660 [gr-qc]].

[4] R. M. Wald, In *Iyer, B.R. (ed.) et al.: Black holes, gravitational radiation and the universe* 69-85 grqc/9710068.

[5] V. E. Hubeny, Phys. Rev. D 59, 064013 (1999) grqc/9808043.

[6] T. Jacobson and T. P. Sotiriou, Phys. Rev. Lett. 103, 141101 (2009) [Erratum-ibid. 103, 209903 (2009)] arXiv:0907.4146 [gr-qc]].

[7] S. Gao and Y. Zhang, Phys. Rev. D 87, 044028 (2013) arXiv:1211.2631 [gr-qc]].

[8] G. Z. Toth, Gen. Rel. Grav. 44, 2019 (2012) arXiv:1112.2382 [gr-qc]].

[9] S. Isoyama, N. Sago and T. Tanaka, Phys. Rev. D 84, 124024 (2011) arXiv:1108.6207 [gr-qc]]; P. Zimmerman,
I. Vega, E. Poisson and R. Haas, arXiv:1211.3889 [gr-qc]. [10] E. Barausse, V. Cardoso and G. Khanna, Phys. Rev. Lett. 105, 261102 (2010) arXiv:1008.5159 [gr-qc]]; E. Barausse, V. Cardoso and G. Khanna, Phys. Rev. D 84, 104006 (2011) arXiv:1106.1692 [gr-qc]].

[11] T. Harada and K. -i. Nakao, Phys. Rev. D 70, 041501 (2004) gr-qc/0407034; E. G. Gimon and P. Horava, Phys. Lett. B 672, 299 (2009) arXiv:0706.2873 [hepth]]; S.-N. Zhang, Int. J. Mod. Phys. D 20, 1891 (2011) arXiv:1003.1359 [gr-qc]].

[12] J. M. Bardeen, in Conference Proceedings of GR5 (Tbilisi, USSR, 1968), p. 174.

[13] E. Ayon-Beato and A. Garcia, Phys. Lett. B 493, 149 (2000) gr-qc/0009077.

[14] S. A. Hayward, Phys. Rev. Lett. 96, 031103 (2006) grqc/0506126.

[15] C. Bambi and L. Modesto, Phys. Lett. B 721, 329 (2013) arXiv:1302.6075 [gr-qc]].

[16] J. M. Bardeen, Nature 226, 64 (1970); C. Bambi, Europhys. Lett. 94, 50002 (2011) arXiv:1101.1364 [gr-qc]]; C. Bambi, JCAP 1105, 009 (2011) arXiv:1103.5135 [gr-qc]]; Z. Li and C. Bambi, JCAP 1303, 031 (2013) arXiv:1212.5848 [gr-qc]].

[17] S. W. Hawking, Phys. Rev. Lett. 26, 1344 (1971). 

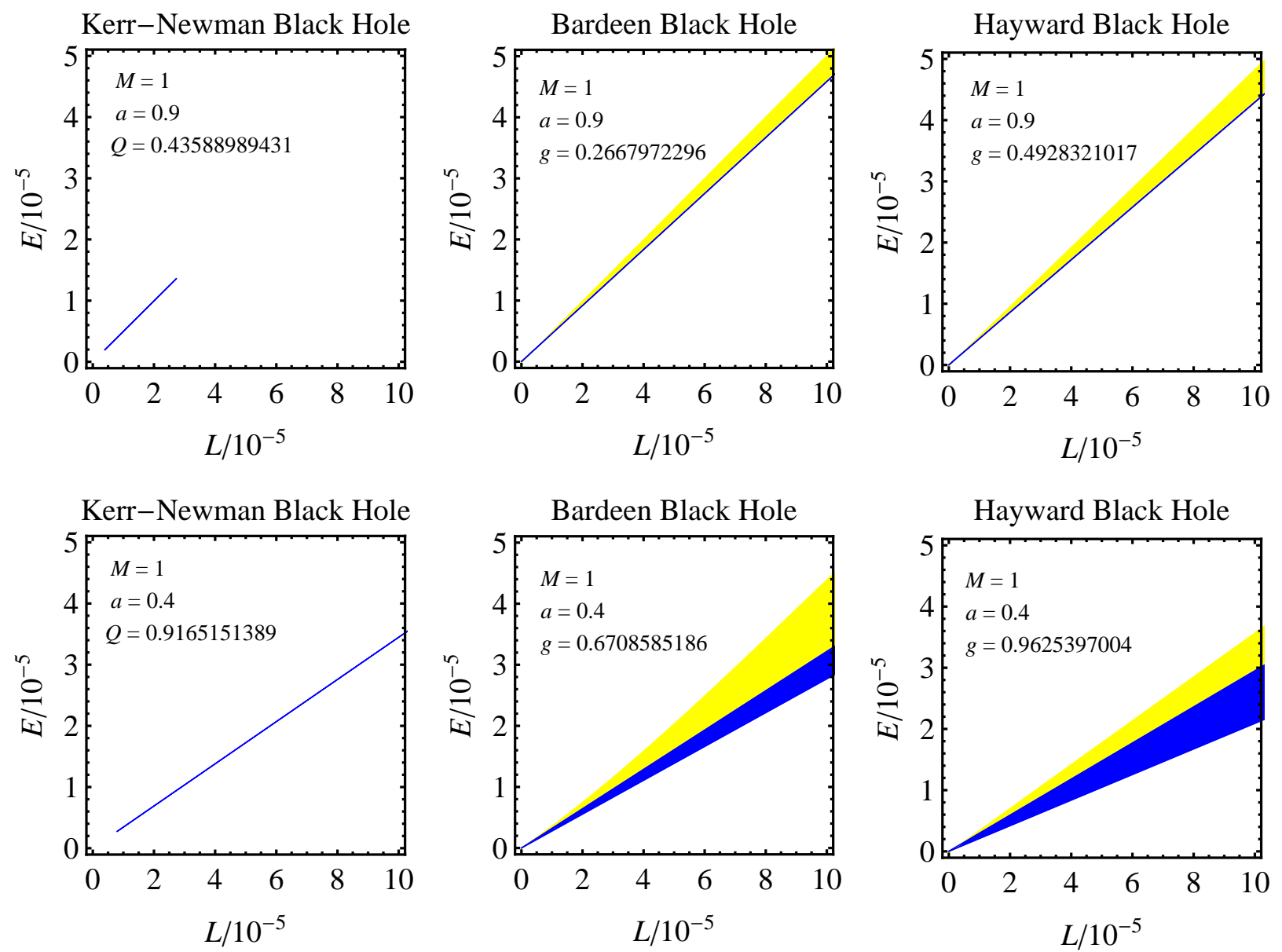

FIG. 1. Values of the energy $E$ and of the angular momentum $L$ for which a test particle can destroy the black hole. Left panels: Kerr-Newman black holes. Central panels: Bardeen black holes. Right panels: Hayward black holes. For regular black holes, the blue-dark region is for bound orbits, the yellow-light one is for unbound orbits. See the text for details.
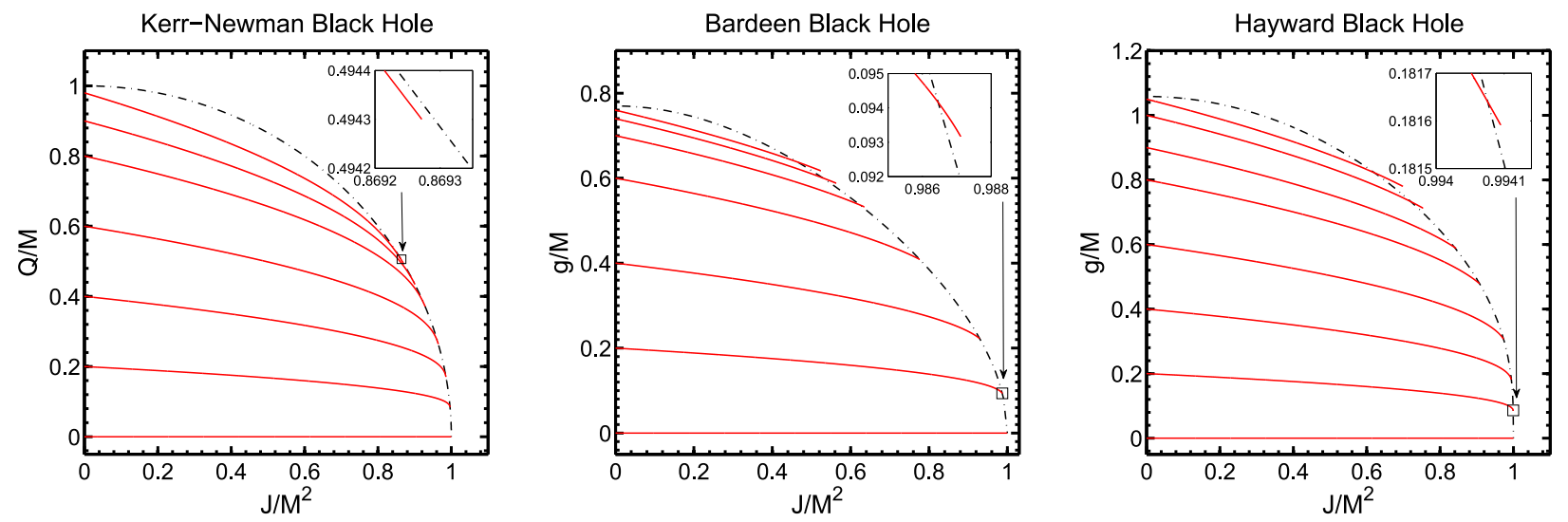

FIG. 2. Tracks of the evolution of Kerr-Newman black holes (left panel), Bardeen black holes (central panel), and Hayward black holes (right panel) as a consequence of the accretion process from a thin disk of uncharged gas. The black dashed-dotted line is the boundary separating black holes and horizonless states. The red curves cannot cross this boundary in the case of Kerr-Newman black holes (the accretion process cannot destroy the black hole), while they can do it in the case of regular black holes (the accretion process can destroy the black hole). See the text for details. 